\title{
A Survey on Hybrid Optical Amplifiers
}

\section{Poda Ramanjaneyulu* and Rajan Miglani}

Department of Electronics and Communication, Lovely Professional University, Punjab, India; anjipoda@gmail.com, rajan.16957@|pu.co.in

\section{Abstract}

The optical fiber communication gains wide attraction in present time. With this communication technique, information can be sent with high data rates and low cost equipment. As there are various advantages of optical amplifier communication but its performance reduces by light dispersion effects. At the receiver end we use various types of optical amplifiers which can amplify these signals. In this paper we review that the hybrid optical amplifiers are combination of one or more amplifiers to remove the drawbacks of individual amplifiers.

Keywords: EDFA Amplifier, Hybrid Amplifiers, RAMAN Amplifier, SOA Amplifier

\section{Introduction}

In communication system the amplifier optical fiber communication is widely used now days because of its properties to provide higher data rates, its low cost etc. In optical fiber communication the light is used as carries and it transmit through thin fiber like structures made from glass (silica) ${ }^{1}$. The light is provided by various sources like: Light Emitting Diodes (LED) and laser. If the optical moves with the Standard Signal Mode Fiber (SSMF) it gets dissipate with the fiber and speed of data is high enough (>15 Gb/s), it get change due to diffraction dispersions. To stop these dissipation we use Optical Fiber Amplifiers (OFA) are used. After the OFA the system has problems like amplified sudden emission. As the signal goes increases the ASE noise will gather the OFA. The bandwidth of the optical fiber is great if the S-band, C-band and L-band are utilized properly. so that optical amplifiers are designed to amplify the signal with the fiber, there must be more gain, more distance from end to end amplifier as long as signal not deform due to high optical power. To make use of the bandwidth we use the Dense
Wavelength Division Multiplexing DWDM and each of the amplifiers must have different bandwidth (Figure 1).

Basically, there are three main elements in fiber optic communication system ${ }^{2}$. They are Compact light source, Low less Optical fiber and Photo detector.

Depending on the application like local area network these light source are required. In this they include power, speed, noise, etc. light emitting diode and laser diode are the main components used in the light sources

Optical fiber well known as the cylindrical dielectric waveguide which is made up of low loss material. It is made up of high quality extruded glass and it is flexible the diameter of this cable in 0.25 to $0.5 \mathrm{~mm}$

The main principle of photo detector is to convert light signal to electrical signal. Depending on the user wavelength PN photo diode and avalanche photo diode is used

\section{Hybrid Amplifiers}

An optical amplifier is a device which amplifies the signal directly without converting into the electrical signals.

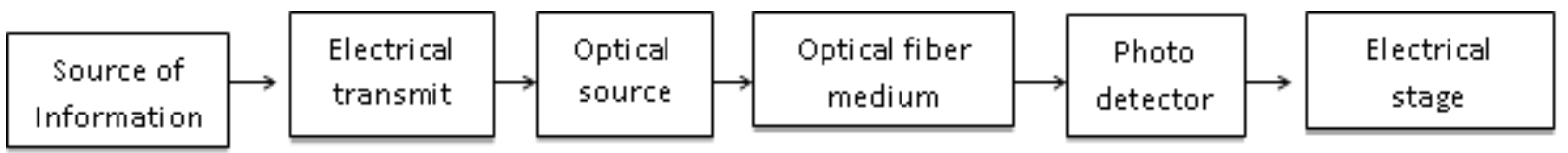

Figure 1. Basic block diagram of optical fiber communication.

${ }^{*}$ Author for correspondence 
This works on the principle of stimulated emission. Stimulated emission is a process where an incoming photon of a specific frequency can interact with the atomic electron causing to drop a lower energy level.Some of the Amplifiers are discussed below

\subsection{EDFA}

Erbium doped fiber amplifier consists of three basic components: length of EDF, wavelength selective coupler and pump laser. The fibre length used depends upon the pump potential, input significance and pumping wavelength. These EDFA can be used large in size in optical fibre communication system as the compatibility with the optical fibre $^{3}$. EDFA is capable of amplifying all the wavelength ranging from 1500 to $1600 \mathrm{~nm}$.there are only C-band and L- band windows for wavelength they allow data signal to simulate the atoms to replace photon. More EDFA are pumped by lasers with a wavelength of either $980 \mathrm{~nm}$ or 1450nm (Figure 2).

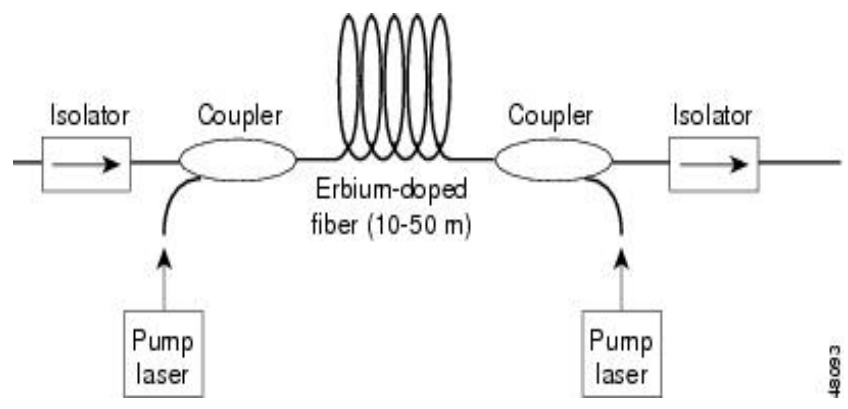

Figure 2. Block diagram of EDFA amplifier.

Demerit of EDFA is different gain for different wavelength due to doping concentrate and EYCDF suppresses concentric quenching affect results in higher gain with flatness under pump power" ${ }^{4}$. Different applications for the EDFA amplifier are shown in Figure 3.

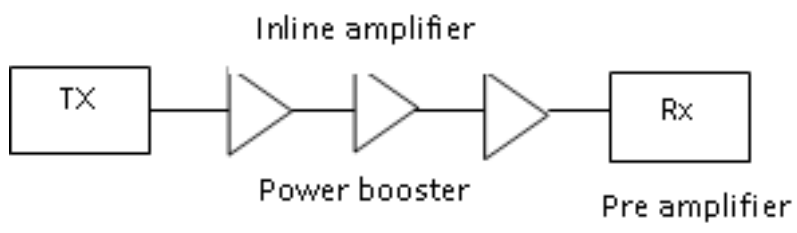

Figure 3. Application diagram of EDFA amplifier.

\subsection{Raman Amplification}

Raman amplifier based upon the gain which results from the effect of stimulated Raman scattering. Raman ampli- fier is little high power pump laser when compared to other amplifiers and wavelength division multiplexing WDM. Here the amplification occur itself in the fibre and it is distributed in the path it has a wide bandwidth of $10 \mathrm{~nm}$.It can amplify up to $10 \mathrm{db}$ in optical fiber. Raman amplifier is combined with EDFA to expand gain flattened bandwidth. Raman gain in optical fibre occur from the transfer of power from one optical beam to another through the energy of photon .If we insert an optical beam in the optical fibre then the Raman scattering occur in an silica glass then the signal passes through the fibre and the frequency around it shift through pump ${ }^{5}$. Raman amplifier based on two cases Raman amplifier distributed and discrete. Here the amplification occurs according to the fibre between two stations and it is near the transmitter then it is forwarded pump or if it is near to the receiver it is reverse pump (Figure 4).

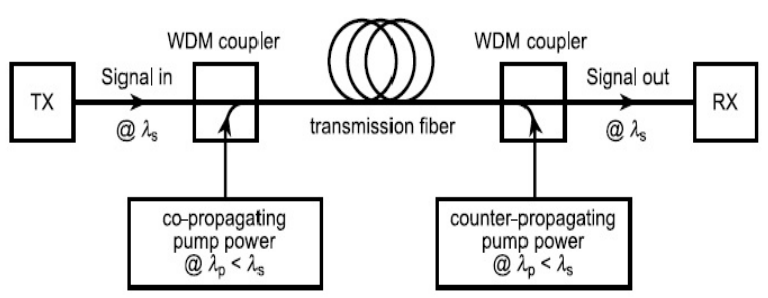

Figure 4. Diagram of Raman amplifier.

\subsection{Semiconductor Optical Amplifier}

Semiconductor optical amplifiers are typically used as a power booster for the optical amplifier and it provide amplification for long distance communication. Semiconductor optical amplifier is a modified semiconductor laser which has different reflectivity and different wavelength. When weak signals are transmitter to the active region of which is stimulate emission result in strong signal emitted from semiconductor (Figure 5).

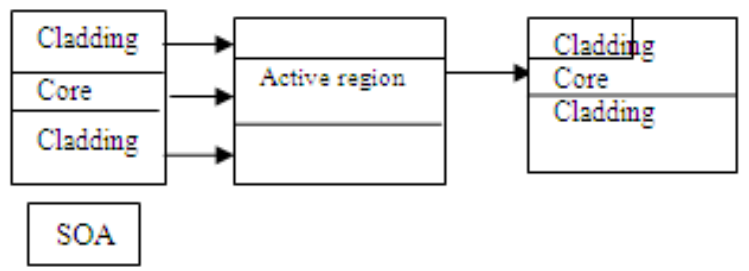

Figure 5. Diagram of SOA amplifier.

Drawbacks of semiconductor optical amplifier high coupling loss polarization dependence a higher noise they 
are typically used for construction of the small package and they work or 1310 and $1550 \mathrm{~nm}^{\text {system }}{ }^{6}$.

\section{Literature Review on Hybrid Amplifiers}

Using the Er-Yb co-doped amplifier we have proposed the flat gain optical gain hybrid amplifier ${ }^{7}$. The contribution for this paper is to implement the flat gain hybrid amplifier based upon the cheapest standard optical amplifiers. After the investigation of the various hybrid amplifiers of dense wavelength and division multiplexing, observed EDFA-Raman amplifier is the best combination for the better results. It is observed that the Raman amplifier is used as a booster for the hybrid distributed Raman amplifier and erbium doped filters. Due to the dynamics gain in the hybrid amplifier this distortion of the pulse shape and cross talk between the symbols presents. The results in terms of the gain flatness, cost brings the good performance and will be suitable for the next generation for the DWDM network.

In this paper we are going to investigate the gain spectrum of a gain clamped amplifier under the various operating conditions and wavelength must be stabilized $\stackrel{8}{\text {. }}$ Mach-zehnder is used to equal the gain clamped and immune the signal to the input where the power in the range of WDM technologies. Due to the high optical gain and value the realization of ideal gain flattered spectrum with fixed gain independent to operating industries clamping and gain flattered operates within in the range of $70 \mathrm{~nm}$ spectral to $1555 \mathrm{~nm}$ using these mach-zehnder equalizer.

Four-Node metro division wavelength and dense multiplexing ring network where the lambda are source at network node and lambda is considered as the virtual ring-. Here the downstream and the upstream for bit error rate is find out using the linbo3neai. Here we are demonstrating four node metro WDM ring network in which all the wavelength are sourced to one node and each wavelength can be sorted at a distinct virtual ring network modulated upstream signal exhibit negligible power penalty both the axis node and the end station are configured to benefit from the component integration

Flat gain L-band propose hybrid optical amplifier for the dense wavelength division DWM ${ }^{10}$ Multiplexing using the two stages of DRA EDFA hybrid amplifiers we have proposed the flat gain corrections. It is observed that if we increase the input power the gain variations over the bandwidth also increases and it is observed from the investigation of DWM system with the $25 \mathrm{Ghz}$ channels and it is observed that all the smooth output spectrum is obtained when all the input power is at $3 \mathrm{mw}$ and it is determined without using any flattering techniques.

\section{Future Scope on Hybrid Amplifiers}

In this paper, we have explained about different types of hybrid amplifiers which are combination of Raman amplifier and EDFA and for others amplifiers it can be extended with gain media. In future there is also a scope for increasing the performance of hybrid amplifiers for the combination of two or more amplifiers.

\section{Conclusion}

According to this study, we have presented a technique which will increase the gain of the amplifier by reducing the attenuation and loss of the signal strength. By adopting various types of hybrid optical amplifiers like EDFA, Raman amplifier and SOA best performance can be achieved when compared to the existing techniques. Hybrid amplifiers have proven effective in DWDM systems to increase long distance having efficient band width with nonlinear effects

\section{References}

1. Mahmud wasfi. Optical Amplifiers Review, International Journal of Communication Networks and Information Security (IJCNIS). 1(1): 2009 Apr.

2. Internet Sources Wikipedia. Different Types of Elements of Optical Communication.

3. Simranjit Singh, Kaler RS. Performance Evolution of Hybrid Optical Amplifiers in WDM Systems, June 2010.

4. Prince Jain, Kadam Vashist, Neena Gupta. Comparison Study of Hybrid Optical Amplifiers (IJSRET), 2014 Dec.

5. Mohammed Syuttaimi, Rahman AB. Review and Performance Limitation Parameters in Optical Amplifiers, 2014.

6. Biswanth Mukherjee. Optical WDM Networks. Springer New York, 2006.

7. Simranjit Singh, Kaler RS. Novel Optical Flat Gain Hybrid Amplifier for DWDM System, 2014 Jan.

8. Yuichi Takushima, Kazuro Kikuchi. Gain Spectrum Equalization of All-Optical Gain-Clamped Erbium-Doped Fiber Amplifier, 1999 Feb. 
9. Reichmann KC, Iannone PP, Birk M, Frigo NJ, Barbier D, Cassagnettes C, Garret T, Verlucco A, Perrier S, Philipsen J. An Eight-Wavelength 160-km Transparent Metro WDM Ring Network Featuring Cascaded Erbium-Doped Waveguide Amplifiers, 2001 Oct.
10. Singh S, Kaler RS. Flat Gain L-band Raman-EDFA Hybrid Optical Amplifier for Dense Wavelength Division Multiplexed System, 2013 Feb. 